\title{
The Earth's Magnetic Field for I922.
}

\author{
By Dr. Louis A. Bauer.
}

THE, precise constitution of the earth's magnetic field at any one time, and the causes of the constituent fields, are problems of fascinating interest, the solution of which appears destined to reveal hitherto unknown properties of matter. A most intimate knowledge of the earth's magnetic and electric phenomena, as well as a thorough acquaintance with all the latest developments of theoretical physics, seems requisite to success in the proper interpretation of the mysteries presented. Whatever theory is advanced, either for the earth's magnetic or its electric field, a hypothesis must be introduced somewhere implying new properties of matter, or changes in the classical laws of electro-dynamics, or physical conditions below or above the earth's surface, of which we have no knowledge at present. This being so, it behoves us to keep an open mind with regard to any new magnetic or electric phenomena which may come to light.

We fortunately have now three bodies, vastly differing from one another in their physical constitution, the magnetic and electric fields of which may come within the scope of our investigations and help us in our theoretical views, namely - the earth, the atmosphere, and the sun. To anticipate, we now know that the direction of the magnetic axis of each one of these bodies is related in the same way for all three to the direction of rotation of the body, and that the magnetic axis of each is inclined to the axis of rotation, namely, at present, about $I r \cdot 5^{\circ}$ for the earth, about $14^{\circ}$ for the atmosphere, and about $6^{\circ}$ for the sun. If $f$ be the physical factor, $\omega$ the angular velocity of rotation, $r$ the radius, and $D$ the density of the body, then the strength of the magnetic fields of these three bodies, at their magnetic poles for example, may be expressed approximately by a formula of the following type:

$$
\mathrm{F}=f . \omega r^{2} \mathrm{D} \text {. }
$$

The magnetic field expressed by (I) has thus far defied laboratory detection, because of the size and speed of rotation of bodies we may experiment with, but it becomes readily appreciable when we are dealing with a body of mass, size, and angular velocity of rotation comparable with those of a member of our solar system. If (I) holds universally, Jupiter, for example, would be enveloped by a magnetic field of about the same strength as that of the sun. Thus we may have to look for assistance in making notable advances concerning the structure and properties of matter to experiments performed by Nature at large.

The chief questions pertaining to the earth's magnetic field may be stated as follows: (a) Is any appreciable portion of the magnetic force observed on the earth's surface to be referred to a non-potential system $\mathrm{N}$ ? (b) Is there, besides an internal magnetic potential system, I, also an appreciable external magnetic potential system, $\mathrm{E}$, existing in our atmosphere? (c) If measurable $\mathrm{N}$ - and E-systems are disclosed, may any portions arise from relativity effects, $R$ ? (d) Is the integral of $d \mu$ over the earth's surface and for all constituent systems equal to zero, where $d \mu$ represents the elemental quantity of magnetism, or any other corresponding physical quantity that may evoke a magnetic field? (e) What physical conditions must the causes for the various systems fulfil to account for the geographic variations, the secular and other variations?

Any theory of the earth's magnetism and electricity will have to give a complete and satisfying account of these various questions before it can be accepted. Doubtless for some time to come we shall have to be content with trying out working hypotheses and must not permit ourselves to be bound to any one theory. However, encouraging progress has been made, and the object of the present communication is to tell of answers more or less complete to some of our questions.

While the magnetic survey of the globe has been in progress by the Carnegie Institution of Washington and other organisations during the past eighteen years, the writer has published the results of various investigations preparatory to a rigorous and complete analysis of the earth's magnetic field. It thus became possible to decide in what regions of the earth the field work should be intensified, and what additional allied scientific data should be included in the observational programme. Furthermore, to satisfy practical demands for magnetic data, our observational work was so arranged that sufficiently accurate results for magnetic charts could be supplied to leading hydrographic establishments within a few months after the observations had actually been made. Thus for the I922 magnetic charts of the British Admiralty, as constructed at the Greenwich Observatory, Sir Frank Dyson states that all available material was used, "the greatest source being the observations made by the Carnegie and the land observations of the Carnegie Institution of Washington."

Pending more accurate and complete reduction of all observations to a common epoch by the Department of Terrestrial Magnetism than was possible by Greenwich Observatory, a preliminary analysis of the earth's magnetic field for I922, on the basis of the latest charts and observations, was made by the writer, with the assistance of various members of his staff. The chief results were announced in a lecture entitled "The Greater Problems of the Earth's Magnetism and their Bearings on Astronomy, Geology, and Physics," delivered at the Carnegie Institution of Washington on November 2I, I922. Some later results were also presented at the meetings of the American Physical Society and the American Astronomical Society at Boston on December 30 and 31 , I 922. ${ }^{1}$ The analysis was made free as possible from assumptions as to the systems composing the entire magnetic field, and was restricted, for the time being, to the region of the earth ( 86 per cent.) between $60^{\circ} \mathrm{N}$. Lat. and $60^{\circ} \mathrm{S}$. Lat. Treating the earth as a spheroid of revolution, spherical harmonic series to the sixth degree, and in some cases to the seventh, were established separately for each of the rectangular components: $\mathrm{X}$, positive towards north; Y, positive towards east; and $Z$, positive towards nadir.

The magnetic data utilised apply in general to longitude-intervals of $10^{\circ}$ and latitude-intervals of $5^{\circ}$. Before the polar caps may be safely included in the analysis, the available magnetic data for these regions will require careful examination, and it may be found necessary to await additional data. Some analyses were also made for the regions $30^{\circ} \mathrm{N}$. Lat. to $30^{\circ} \mathrm{S}$. Lat., and $45^{\circ} \mathrm{N}$. Lat. to $45^{\circ} \mathrm{S}$. Lat. There is evidence that the Gaussian coefficients defining the earth's magnetic field, are to be regarded at first as purely empirical quantities and, hence, strictly applicable only to the region of the earth from which they were derived. It would seem, however, that inclusion of the polar caps will not materially alter the main conclusions given here.

The chief conclusions from our analysis are as follows : ${ }^{2}$

I. For a satisfactory representation of the observed data, it is necessary to recognise the existence of an

${ }^{1}$ Phys. Rev., March 1923, pp. 370-37x and 388; also Pop. Astr., March 1923, p. 186 and Atm. Elect. for March-June (pp. r-28), and September 1923 . 
internal magnetic system, I, an external system, E, and a non-potential system, $N$, or of three equivalent systems, X, Y, Z. The I-system constitutes about 94 per cent. of the total magnetic field, and $\mathrm{E}$ and $\mathrm{N}$, each about 3 per cent. (There is a possibility that relativity effects, $R$, may play a part in the exact evaluation of the three systems.)

2. As a resultant effect of all systems causing the secular variation of the earth's magnetism, the north end of the magnetic axis of the I-system during the past eighty years has been moving slowly towards the west, and apparently at the same time slowly towards the equator. The indications from all available data are that if the magnetic axis completely revolves around the axis of rotation, regarding the possibility of which there may be some doubt, the period would not be some hundreds of years, but many thousands of years. The magnetic secular variation results from changes, with lapse of time, both in the direction of magnetisation and in the intensity of magnetisation ; the latter quantity has been steadily diminishing during the past eighty years at the annual average rate of about I/I 500 part.

3. A suggestive effect, dependent apparently upon the distribution of land and water, has been disclosed, namely, that the average equivalent intensity of magnetisation for corresponding parallels north and south, is generally larger for the land-predominating parallel than for the ocean-predominating parallel. The secular changes, however, are on the average larger per annum for the south, or ocean-predominating, hemisphere than for the north, or land-predominating, hemisphere.

4. For the earth's internal uniform magnetic field, the following data apply for r 922 . The magnetic moment, M, is $8.04 \times 10^{25}$ C.G.S. $;^{3}$ the components of $M$, respectively parallel and perpendicular to the earth's axis of rotation, are, $\mathrm{M}_{p}=7.88 \times \mathrm{IO}^{25}$ C.G.S., and $\mathrm{M}_{e}=\mathrm{I} \cdot 60 \times \mathrm{IO}^{25}$ C.G.S. ; $\mathrm{M}_{p}=4 \cdot 93 \mathrm{M}_{e}$. Were the earth's magnetism uniformly distributed throughout the earth, which is not likely, the average intensity of magnetisation would be 0.074 C.G.S. The magnetic axis intersects the North Hemisphere in latitude $78^{\circ} 32^{\prime}$ North and longitude $69^{\circ} \mathrm{o} 8^{\prime}$ West of Greenwich.

There has recently appeared an account of an analysis of the earth's magnetic field, also for I922, by Sir Frank Dyson and Prof. H. H. Turner. These authors reach conclusions which apparently are at variance with mine given in (I) as to the definite existence of the two systems, $\mathrm{E}$ and N. However, a critical examination of the residuals obtained by them when they endeavour to represent the rectangular components, $\mathrm{X}, \mathrm{Y}, \mathrm{Z}$, on the hypothesis of a magnetic potential due alone to systems below the earth's surface, and assuming that a non-potential system does not exist, is found, in fact, to strengthen my conclusions. ${ }^{5}$

Question (a) (Non-potential System, N).-The existence of the $\mathrm{N}$-system implies the non-vanishing of the line integral of the magnetic force taken around a closed circuit on the earth's surface. Such line integrals have been computed for large land areas, like the United States, and for very large ocean areas, with data from the cruises of the Carnegie, both in the Northern and Southern Hemispheres. The results are so consistent that they cannot be accounted for wholly by observational errors. The analysis of the earth's magnetic field shows that the coefficients derived from the east-west component, $Y$, will not give an entirely satisfactory representation of the south-

a The value of the magnetic moment frequently found in text-books, as dependent on Gauss's analysis for 1830 , is $8.55 \times 10^{25}$ C.G.S. The average annual rate of loss between $I 830$ and $I 922$ is about I/I500 part, thus corresponding with the annual average rate as given in $(2)$

1 Mon. Not. Roy. Astr. Soc., Geophys. Sup., vol. i. No. 3, May r923, pp. $76-88$.

Terr Mag. and Atm. Elect. for March-June 1923, pp. 24-28. north component, X. A similar experience has been encountered in recent analyses of the diurnal variation of the earth's magnetic field, of magnetic disturbances, and of eclipse effects. Thus the evidence is in favour of the existence of non-potential magnetic systems. The difficulty has been in the adequate physical interpretation of the results. According to classical theory, line-integral values are a measure of electric currents passing perpendicularly through the area enclosed by the circuit. The average strength of such indicated currents for the earth's magnetic field is found to be more than ro,ooo times that of the vertical conduction current of atmospheric electricity. The average strength of vertical currents that may in part be responsible for the magnetic diurnal variation is about 2000 times that of the currents causing the diurnal variation of atmospheric electricity. We are then forced to conclude that the magnetic lineintegrals are a measure of something else than is recorded by atmospheric-electric instruments. Various suggestions are at present receiving careful consideration. The very interesting point was recently raised by Sir Arthur Schuster that no one, so far as he knew, had experimentally verified the generally accepted hypothesis that the magnetic force was accurately at right angles to the current which produced it, and he further remarked that he had very recently come across the statement that according to Einstein's theory the force and the current should not be exactly at right angles. But there are at present difficulties in trying to attribute the observed non-potential effects wholly to such a possible relativity cause.

The general system of vertical currents for the earth's field is as follows: negative electricity flowing into the earth in polar regions and flowing out in lower latitudes; for positive electricity these directions would of course be reversed. The system of vertical currents is unsymmetrical both about the axis of rotation and the equator. (A similar system of vertical currents will explain the present facts of the annual variation of atmospheric electricity.) Enough has been said to show of what extreme interest the final elucidation of the magnetic non-potential effect is likely to be.

Question (b) (External Potential System, E).-This system is disclosed by the fact that the coefficients determined from the horizontal components, $\mathrm{X}$ and $\mathrm{Y}$, will not reproduce completely the vertical component, $Z$, but will leave outstanding effects of a character which, according to classical theory, can only be explained by an external system of electric or magnetic forces. However, if any portion of the earth's total magnetic field is to be attributed to causes which involve relativity effects, $R$, then $E$, in whole or in part, may have to be regarded as resulting from $R$. It is hoped that a special investigation now under way will throw further light on this interesting question.

As the result, apparently, of the extensive increase in knowledge of the earth's magnetic field over that at the command of previous analysts, the coefficient of the first degree zonal harmonic is found to be three times that resulting from Schmidt's careful analysis for I885. The magnitudes of Schmidt's coefficients for the various zonal harmonics were such that. he did not deem it safe to draw a definite conclusion as to the reality of an external system. The case is different, however, for our 1922 analysis; as stated under conclusion ( $\mathrm{I}$ ), we can no longer ignore the existence of effects similar to those from an external system.

Question (c) (Relativity Effects).-This question has already received some attention in the consideration of questions $(a)$ and $(b)$; it also enters into question $(d)$.

Question (d) (Is the Integral of $d \mu$ Zero?).-All analysts beginning with Gauss have assumed that the integral of $d \mu$ is zero. The assumption enters not only into the determination of the coefficients of the 
$Z$-series, but may also require consideration in the derivation of the coefficients of the $\mathrm{X}$-series and of the $Z$-series, if there is a non-potential system $N$. So far as the $Z$-component is concerned, if we do not assume the integral to be zero, a small constant term is added to the $Z$-series, which slightly improves the mathematical representation. If we have an $\mathrm{N}$ system caused by vertical currents, as already described, then the question arises whether for a limited portion of the earth, for example from $60^{\circ} \mathrm{N}$. Lat. to $60^{\circ} \mathrm{S}$. Lat., we may legitimately assume that the total amount of electricity leaving the earth equals the total amount entering it in this region ; if not, then $\int d \mu$ would not be exactly zero. It is of interest to note that Gauss himself intimated, in his celebrated memoir on the "General Theory of the Earth's Magnetism," that the day might come when it could not longer be assumed that the integral of $d \mu$ is zero. Investigations in progress will further elucidate this matter.

Question (e) (Variations of the Earth's Magnetic Field). - We now come to crucial tests that may be applied to any theory of the cause of the earth's magnetic field. It would seem as though the surest approach to a solution of the two problems, the origin of the earth's magnetic field and the origin of the earth's electric field, will be by means of the striking variations, geographic, diurnal, annual, sun-spot, and secular, to which they are subject. The two chief sets of variations, which a theory of the earth's magnetic field will have to explain satisfactorily, are: (I) the geographic variations; (2) the secular variations.

Fig. I is intended to show how $\rho$, the equivalent intensity of magnetisation or any other corresponding physical quantity, would have to vary from parallel to parallel in order to produce the portion (about 70 per cent.) of the earth's total magnetic field symmetrical about the axis of rotation, as represented by zonal harmonics to the sixth degree inclusive. If this portion of the field were uniform, then $\rho$, represented by the radius-vector from $\mathrm{O}$, would be constant; this case is shown by the outer circle. Were the zonal field symmetrical about the equator, then instead of the outer circle we have an ellipse, which has been drawn for each of the two epochs I 885 and 1922 (indicated by broken curves); for this case $\rho$ for the equator would be about I 7 per cent. greater than for the combined parallels $60^{\circ} \mathrm{N}$. and S. The heart-shaped full curves represent the actual state of affairs for the field symmetrical about the axis of rotation. Comparing the radii vectores, $\rho$, for corresponding parallels of latitude, north and south, it is seen that for both curves (I885 and I922) $\rho$ is invariably greater for a land-predominating parallel than for an ocean-predominating parallel, and this fact obtains even for the dotted portions of the curves which apply to the polar regions (see conclusion 3.) It will be noticed that the 1922 heartshaped curve lies wholly within the 1885 one, just as was the case for the ellipses, and the difference, $d \rho$, between the curves represents, proportionately, the shrinkage in the earth's magnetic moment, or in the equivalent intensity of magnetisation, between I 885 and I922. It will be noticed that the shrinkage is greater for the south, or ocean-predominating, hemisphere, than for the north, or land-predominating, hemisphere. The effect of the distribution of land and water is one calling for careful examination, and its further study may result in material advancement of our knowledge as to the cause or causes of the earth's magnetic field.

If we wish also to take into account the balance of the earth's magnetic field, about 30 per cent., which is unsymmetrical about the axis of rotation and is represented by the tesseral harmonics, then the pearshaped solid, obtained by the revolution of the heart-shaped curve about the earth's axis of rotation, 
quite comparable with that of well-seasoned magnets. The loss occurs practically all in the uniform portion of the earth's magnetic field, parallel to a diameter, inclined at present to the axis of rotation about II. $5^{\circ}$; the loss is not made up by any material gain in the non-uniform, heterogeneous portion of the earth's magnetic field. ${ }^{8}$ The annual percentage loss is nearly the same for the polar and equatorial components of the uniform magnetic field; and as the equatorial component is only about one-fifth that of the polar component, the absolute annual loss in the earth's magnetic moment results almost entirely from the polar component, i.e. the uniform portion of the earth's magnetic field, symmetrical about the earth's axis of rotation, suffers nearly the entire loss.

The system of forces which must be superposed upon the uniform internal magnetic field of 1885 in

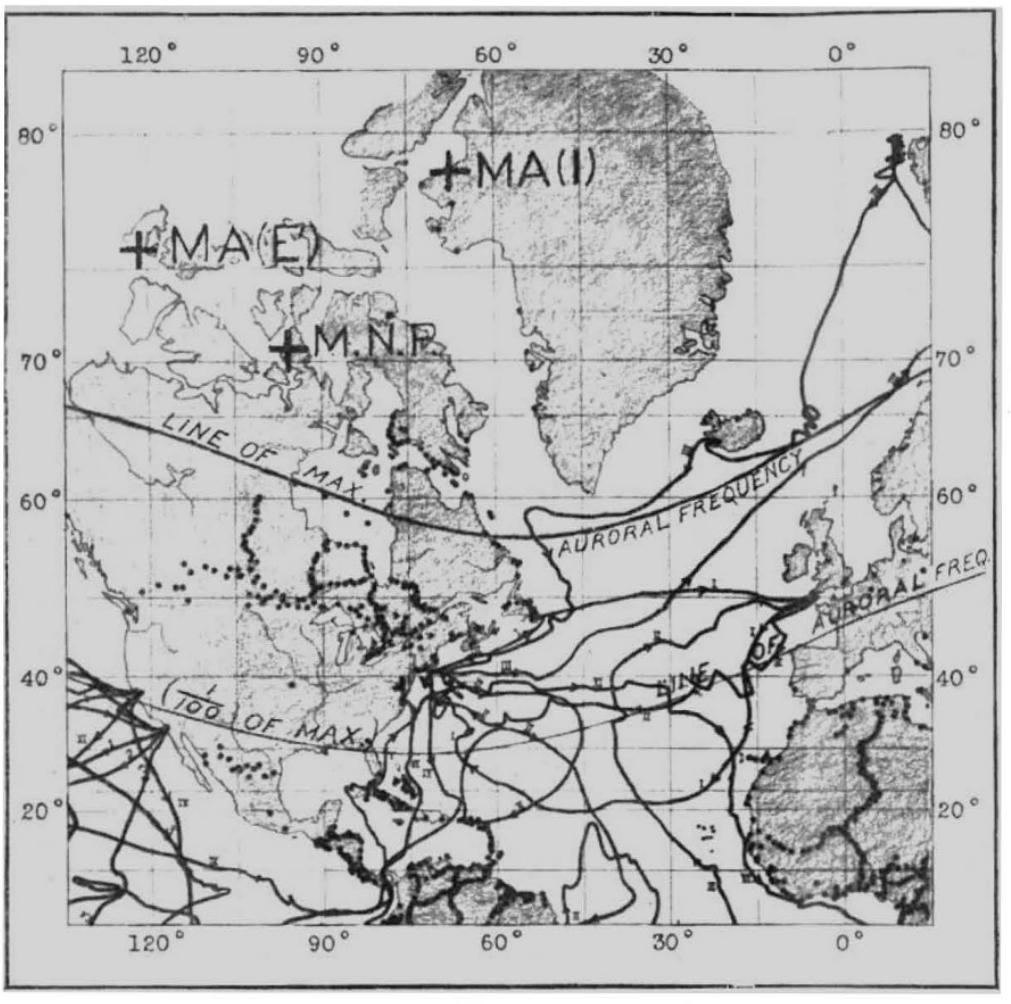

Fig. 2.

order to obtain the observed field for 1922 proves to be a demagnetising system, the magnetic axis of which is directed almost diametrically opposite to that of the primary uniform field. A similar result was found in $1904^{9}$ for the period I890-1900. In brief, the secular-variation system shows the characteristics of the self-induced field of a uniformly magnetised body.

Let us next inquire briefly into which of the quantities in formula (I) so large an annual rate of change for the earth's field as I/I5Oo part is to be attributed? Certainly not to the angular velocity, $\omega$, or to the radius, $r$, or to the density, $\mathrm{D}$, since changes on the order of $I / I_{5} 00$ part in one of these quantities, or in their combined product, would not escape detection by other means. We must conclude that the physical factor, $f$, contains within itself the kernel for the observed change, but what does this imply?

Let us suppose next that in the factor $f$ we have embodied some physical relation upon which both the earth's magnetic field and its gravitational field

- Terr. Mag. and Atm. Elect., vol. 8 (r903), p. roy, and vol. 28 (r923), p. 2 r. - Terr. Mag. and Atm. Elect., vol. 9 (1904), pp. 181-186. depend. Then on the basis of the large average annual loss during the past eighty years in the strength of the earth's magnetic field, we can immediately say that magnetism and gravity are not related to each other as the first power of the factor, for otherwise a correspondingly large annual change in gravity would likewise have been observed. Again, while gravity is greater over the oceans than over continents, the equivalent intensity of magnetisation is, on the average, somewhat less for ocean areas than for continental areas, so here again there is no immediate relation between gravity and magnetism. According to Sutherland's theory ${ }^{10}$ which was based on a slight modification of the laws of electrodynamics, magnetism would depend on the first power of a small fraction $\beta$ (about $2.6 \times \mathrm{IO}^{-22}$ ), and gravity upon the second power ; this quantity $\beta$ would enter into the factor $f$ of formula ( $\mathrm{I}$ ). Accordingly the annual decrease of $I / I 500$ part in magnetism would imply, on Sutherland's theory, only a decrease of the square of I/I 500 part, or about one-half of a millionth part in gravity, and this is a quantity which may readily escape detection with our present gravity appliances, unless the accumulative effect over many years be carefully observed at several standard stations. Hence, a theory involving gravity and magnetism in the manner prescribed by Sutherland's hypothesis might be admissible. But the observed decrease in the earth's magnetic field-strength would then have to be referred to a corresponding change in $\beta$. But what makes $\beta$ change ? It was only meant to represent a very slight variation in the law of action between electric charges ; if $\beta$ changes, so must the new assumed law of electro-dynamics. We have under investigation various hypotheses to account for the observed secularchanges in theearth's magnetic field.

Sufficient has been given to show with what extreme care a theory of the earth's magnetic field will have to be formulated and how exhaustively it will have to be examined in the light of the data now known to us. No one who will familiarise himself with the facts will lightly announce the discovery of a new theory of the origin of the earth's magnetism. New and interesting matters may confidently be expected from the discovery of the true cause.

In conclusion, Fig. 2 is presented to show the positions of the following points: MA(I), north end of magnetic axis of the earth's uniform internal magnetic field in I922, latitude $78^{\circ} 32^{\prime}$ N., and longitude $69^{\circ} 08^{\prime}$ W. ; MA(E), north end of magnetic axis of the earth's uniform external magnetic field in 1922 , latitude $76.8^{\circ} \mathrm{N}$. and $12 \mathrm{I} \cdot 4^{\circ} \mathrm{W}$. ; and N.M.P., the approximate position of the North Magnetic Pole in 1904 , latitude $70.5^{\circ} \mathrm{N}$., and longitude $95.5^{\circ} \mathrm{W}$. As will be seen, the line of maximum auroral frequency passes to the south of the three positions. (The other lines shown are the routes of the Carnegie.) It will be noticed that the displacement of the E-axis is about $52^{\circ}$ west of that of the I-axis, and that the N.M.P. is about midway in longitude between I and E. From the amount and direction of displacement of the E-axis with reference to the I-axis, we may deduce further important facts bearing upon the theory of the earth's magnetic field and the possible conductivity of interplanetary space.

${ }_{10}$ Terr. Mag. and Atm. Elect., vol. 9 (1904), pp. 167-172. 